\title{
Millimagnitude Optical Photometry for the Transiting Planetary Candidate OGLE-TR-109 ${ }^{1}$
}

\author{
José Miguel Fernández ${ }^{1}$, Dante Minniti ${ }^{1}$, Grzegorz Pietrzynski ${ }^{2,4}$, Wolfgang Gieren ${ }^{2}$, \\ María Teresa Ruíz ${ }^{3}$, Manuela Zoccali ${ }^{1}$, Andrzej Udalski ${ }^{4}$ and, Thomas Szeifert ${ }^{5}$
}

\begin{abstract}
We present precise $V$-band photometry for the low-amplitude transit candidate star OGLE-TR-109. This is an extreme case among the transiting candidates found by the OGLE group because of the early spectral type of the star (F0V), of the low transit amplitude $\left(A_{I}=0.008 \mathrm{mag}\right)$, and of the very short period $(P=0.58909$ days) of the orbiting companion. Using difference image photometry, we are able to achieve millimagnitude errors in the individual data points. One transit of this star is well defined in our light curve. This confirms the OGLE detection and rules out the possibility of a false positive. The measurement of this transit allows to refine the transit amplitude $\left(A_{V}=0.006 \pm 0.001\right.$ mag), and the ephemerides for this interesting system, as well as the radius of the possible orbiting companion $\left(R_{P}=0.90 \pm 0.09 R_{J}\right)$, and the inclination of the orbit $(i=77 \pm 5 \mathrm{deg})$. Two other transits observed at lower $\mathrm{S} / \mathrm{N}$ confirm the period of this system measured by OGLE. There is no evidence for a blend of the F-type main sequence star with a redder eclipsing binary, or for secondary transits in the present observations. The absence of ellipsoidal modulation in the light curve of the primary rules out a low mass star companion or brown dwarf with $M>14 \pm 8 M_{J}$. The remaining possibilities for OGLE-TR-109 are a blend between the F-type star and a binary with a bluer primary star, or a new transiting extrasolar planet.
\end{abstract}

\footnotetext{
${ }^{1}$ Department of Astronomy, Pontificia Universidad Católica, Casilla 306, Santiago 22, Chile

E-mail: dante@astro.puc.cl, jfernand@astro.puc.cl, mzoccali@astro.puc.cl

${ }^{2}$ Department of Physics, Universidad de Concepción, Casilla 160-C, Concepción, Chile

E-mail: pietrzyn@hubble.cfm.udec.cl,wgieren@astro-udec.cl

${ }^{3}$ Department of Astronomy, Universidad de Chile, Santiago, Chile

E-mail: mtruiz@das.uchile.cl

${ }^{4}$ Warsaw University Observatory, Al. Ujazdowskie 4, 00-478 Waszawa, Poland

E-mail: udalski@astrouw.edu.pl

${ }^{5}$ European Southern Observatory, Vitacura, Santiago, Chile

E-mail: tszeifer@eso.org
} 
Subject headings: Stars: individual (OGLE-TR-109) - Extrasolar planets: formation

\section{Introduction}

Udalski et al. (2002) discovered very low amplitude transits in the $V=15.80, I=14.99$ magnitude star OGLE-TR-109, located in the Carina region of the Milky Way disk, at $R A(2000)=10: 53: 40.73, D E C(2000)=-61: 25: 14.8$. They monitored 24 transits, measuring an amplitude $A_{I}=0.008 \mathrm{mag}$, and a period $P=0.58909$ days.

Based on near-infrared photometry and low-dispersion spectroscopy, Gallardo et al. (2005) identify OGLE-TR-109 as an early F-type main sequence star located at a distance $d=2.59 \pm 0.25 \mathrm{kpc}$, with reddening $E(B-V)=0.38 \pm 0.02$. This star is intrinsically 2 magnitudes brighter than the Sun, with $M_{V}=2.55$, and also larger, with $R_{s}=1.5 R_{\odot}$.

OGLE-TR-109 has been target of a few studies due to its remarkable location in the parameter space. It shows a very small transit amplitude as well as very short orbital period, as illustrated in Figure 1. Furthermore the spectral type of the primary is the earliest so far reported for OGLE transits.

Udalski et al. (2002) estimated $R_{s}=1.23 R_{\odot}$ for OGLE-TR-109, and a lower limit of $R_{p}=0.099 R_{\odot}$ for its companion, arguing that the small $R_{p}$ gives a larger probability that this is an extrasolar planet. Upon examining in detail a number of OGLE candidates, Gallardo et al. (2005), and Silva \& Cruz (2005) also considered the star OGLE-TR-109 as a prime planetary candidate. Pont et al. (2005), however, classified it as an unsolved case, pointing out three possibilities: a blend of an F-type star with a background binary, a true planetary transit, or a false positive transit detection. They did not detect radial velocity changes at $\sigma=1.5 \mathrm{~km} / \mathrm{s}$ level, corresponding to an upper companion mass limit of $45 M_{J}$. They argued that the false positive detection is the most likely explanation. In this paper we discard this possibility, because we detect the individual transits beyond doubt.

Estimating the expected binary star ellipsoidal modulations due to tidal effects, Drake (2003) was the first to point out that it would be possible to limit the mass of the secondary using accurate photometry alone, procedure that was further refined by Sirko \& Paczynski (2003). In this paper we use the absence of modulation to show that OGLE-TR-109 (if it is

\footnotetext{
${ }^{1}$ Based on observations collected with the Very Large Telescope at Paranal Observatory (ESO Programme 075.C-0427(A), JMF and DM visiting observers).
} 
not a blend) should have a very low mass companion

Section 2 presents the observations and photometry of OGLE-TR-109. Section 3 explores the likelihood of a planetary companion. Section 4 examines the possibility of a blend with an eclipsing binary. Finally, section 5 gives our main conclusions.

\section{Observations and Data Reduction}

Moutou et al. (2005) demonstrated the high quality photometry that can be obtained with the VLT. They achieved millimagnitude photometry during the transit of OGLE-TR132, refining its amplitude and the planetary parameters. We are carrying out a similar program for several recently discovered OGLE transit candidates, with OGLE-TR-109 being located in one of the four monitored fields.

The photometric observations were taken with VIMOS at the Unit Telescope 4 (UT4) of the European Southern Observatory Very Large Telescope (ESO VLT) at Paranal Observatory during the nights of April 9 to 12, 2005. All four nights were clear throughout, with sub-arcsecond seeing during most of the time. The VIMOS pixel scale is 0.205 arcsec/pixel, and no images were under-sampled during this run. Given the large VIMOS field of view that consists of four $7 \times 8$ arcmin fields covered by the four CCDs arranged in a square pattern with a separation gap of 2 arcmin, we monitored a number of OGLE transit candidates simultaneously. Here we report on the observations of OGLE-TR-109, the other stars will be discussed elsewhere. No standard stars were observed, because we will perform difference photometry.

Typically 150 points per night were obtained in the field of OGLE-TR-109, resulting in well sampled transits. The observations lasted for about 9 hours per night, until the field went below 3 air-masses. Figures 2 and 3 show examples of our best images taken near the zenith, and worst images taken at very high airmass.

We used the Bessell $V$ filter of VIMOS, with $\lambda_{0}=5460 \AA, F W H M=890 \AA$. We choose the $V$-band in order to complement the OGLE light curves which are made with the $I$ band filter. One of the main objectives of this work was to discard blends and binary stars present among the transit candidates. For this, light curves measured in the $V$-band can be compared with the OGLE light curves in the I-band, and non-planetary eclipses can be discarded when very different amplitudes are measured, for example. In addition, while the $I$-band filter is more efficient for transit searches (Peeper \& Gaudi 2005), the $V$-band shows better the effects of limb darkening during the transit, and is adequate for the modeling of the transit parameters. 
In order to reduce the analysis time of the vast dataset acquired with VIMOS, we decided to cut postage stamp images around the transit candidates rather than process the whole images. The future processing of the whole images is nevertheless a gold mine to identify additional transit candidates $\sim 2$ magnitudes fainter than the OGLE search (Fernandez et al. 2006, in preparation). We estimate that the whole images contain $>10000$ stars with $15<V<19$ for which light curves can be obtained with individual photometric errors $<0.01 \mathrm{mag}$.

The images of OGLE-TR-109 analyzed here are $400 \times 400$ pix, or 80 arcsec on a side. Each of these small images contains about 500 stars with $15<V<24$ that can be used in the difference images, and light curve analysis. The 7 best seeing images were selected, and a master image was made for each night. These master images serve as reference for the difference image analysis (see Alard 2000, Alard \& Lupton 1998).

The photometry of OGLE-TR-109, with mean $V=15.80$ gives $r m s=0.002$ to 0.010 mag throughout most of the whole run. Figure 5 shows the full light curve for all four nights, including all data points, which are 650 in total. Indicated are the expected mean times of transits as computed by Udalski et al. (2002). The large scatter at the end of the nights is due to high airmass, and degraded image quality. There the FWHM of the PSF grows to more than twice its original size of about 3 pixels $(0.6$ arcsec $)$. These regions of the light curve will be excluded from the following discussion, although we note that there is evidence for the transit at the end of night 4 , the scatter is too large to measure its duration or amplitude.

Figure 6 shows the light curve along the third night of observations, when the best transit was monitored. Figure 7 shows the phased light curve of the OGLE $I$-band photometry for comparison. Although not devoid of scatter, there are $N_{t}=32$ points in our single transit shown in Figure 6, and the reality of this transit is beyond doubt. For a given photometric precision of a single measurement of $\sigma_{p}$ and a transit depth $A$, Gaudi (2005) gives the following equation to estimate the signal-to-noise:

$$
S / N=N_{t}^{1 / 2} A / \sigma_{p}
$$

For OGLE-TR-109 we find the $\mathrm{S} / \mathrm{N}$ of this transit to be $S / N=17$ using $A=0.006$ and $\sigma_{p}=0.002$. The $S / N$ of the transits during the first and fourth night are poorer $(\sim 5)$ merely due to the larger scatter in the individual photometric points.

The full OGLE photometric dataset for OGLE-TR-109, covering now almost 2000 cycles (40 individual transits) make it possible to significantly refine the ephemerides of this system: 
$H J D($ middle of transit $)=2452322.55993+0.589128 E$

Then, Figures 6 and 7 clearly lead to our first result: that OGLE-TR-109 is not a false positive transit detection. Having ruled out that possibility of a false positive, we consider the remaining three possibilities: a low-mass stellar companion, a planetary transit and a blend with an eclipsing binary star.

\section{A Brown Dwarf?}

Early F-type main sequence stars are potentially interesting for astrobiology because their habitable zones are much larger than those of later type stars like our Sun (Kasting, Whitmire \& Reynolds 1993). Unfortunately, planets have not been discovered in early type F-stars. Planetary transits are more difficult to find (and confirm) in F-type stars than for later type stars, because the transit amplitude decreases with the square of the stellar size. Thus, one expects the typical transit of a Jovian planet to have amplitudes of the order of $A=0.005,0.01$, and 0.02 mags for $\mathrm{F}, \mathrm{G}$, and $\mathrm{K}$-type main sequence stars, respectively. Furthermore, F-type stars have fewer and wider lines in the optical region of the spectrum, and are sometimes rotating very fast, which prevents from achieving the very small radial velocity errors necessary for the detection of planets. Therefore, it is not surprising that in the extrasolar planet lists there is a lack of F-type stars with respect to G and K-type stars. There are twenty F-type stars in the Extrasolar Planets Encyclopedia (Schneider 2005), but none earlier than F7V. Nothing is known about the existence of planets in earlier type stars. Because they are difficult to find, it is important to discover them. In their favor, we should mention that usually F-type stars are intrinsically 1-2 magnitudes brighter that G and K-type dwarfs, and that their larger size allows a wider range of inclination angles for observable transits.

The transit time measured by Udalski et al. (2002), shown in their fit to the phased light curve, lasts $t_{T}=2.1$ hours, which is at odds with the interpretation of a planet transiting in front of a Solar type $\operatorname{star}(\sim 1.5$ hours for $P=0.589)$. This long transit time argues for an over sized star, either a subgiant or an earlier type main sequence star. Gallardo et al. (2005) rules out a subgiant, on the basis of optical and near-infrared photometry and low dispersion spectroscopy. They favor an early F-type main sequence star with $R_{s}=1.52 R_{\odot}$ and $T=7580 \pm 350 \mathrm{~K}$. Pont et al. (2005) obtain similar parameters, with $R_{s}=1.3-1.4 R_{\odot}$ and $T \sim 7000 \mathrm{~K}$ based on their high dispersion, low $\mathrm{S} / \mathrm{N}$ spectra. We adopt the means of the extreme values, $R_{s}=1.4 R_{\odot}$ and $T=7300 \mathrm{~K}$, because they have been independently measured, and there is no reason a priori to favor one over the other. This corresponds to 
an F0V star with mass $M=1.5 M_{\odot}$. Note that a star of this type has a radiative envelope, which does not require a synchronized orbit, in spite of the short orbital period, unless the companion turns out to be very massive (Zahn 1977). On the other hand, circularization of the orbit must have occurred rapidly, so we assume $e=0$ for this system.

Using the full transit observed during the third night (Figure 6), we measure $t_{T}=$ $1.8 \pm 0.1$ hours, which is consistent with $R_{s}=1.4 R_{\odot}$ for $P=0.589$ days. The measured amplitude of the transit was $A_{V}=0.006 \pm 0.001 \mathrm{mag}$.

We used an image of the Sun taken at the Big Bear Solar Observatory on February 2, 2005, as a proxy to treat limb darkening realistically to fit the light curve (Silva \& Cruz 2005). Given the period and the estimated mass of the host star, we were able to constrain the semi-major axis $a$ of the orbit $\left(2.3-2.5 R_{s}\right)$. This short orbit and the star size of $R_{s}=1.4 R_{\odot}$ also determines that the allowed range of inclination angles is relatively large. Transits would occur for $66<i<90$ in this system. In fact, the geometric probability of detecting a transit in a system like this is quite large, $\sim 27 \%$. The inclination of the system is constrained by the duration of the transit. This inclination cannot depart too much from edge-on because the transit is long. The best fit corresponds to a transiting object with $R_{p}=0.90 \pm 0.09 R_{J}$ for our adopted stellar size of $R=1.4 R_{\odot}$, and an inclination of $i=77 \pm 5 \mathrm{deg}$. We measured the transit duration from the best fit, from first to fourth contact.

We searched the best fit minimizing chi-square in the parameter space $\left(R_{p}, a\right.$ and $\left.i\right)$. To calculate the uncertainties, we fixed the values of two parameters and let the third to vary until the change in chi-square showed a confidence corresponding to 3-sigma.

The fit is shown in Figure 8. Figure 9 shows the orbit of OGLE-TR-109 and its transit configuration drawn to scale for different inclination angles: $i=90,84,78,72$, and 66 degrees from top to bottom. The smaller inclinations are ruled out because the measured transit duration is too long for a grazing eclipse, unless the size of the star is much larger, which appears to be ruled out by the optical-IR photometry and spectroscopy (Gallardo et al. 2005).

The amplitude value of $A_{V}=0.006 \pm 0.001 \mathrm{mag}$ is smaller than the amplitude measured by Udalski et al. (2002) for OGLE-TR-109, $A_{I}=0.008$ mag. This is at odds with the expectations, because stellar limb darkening curves in the $V$-band should be shallower in the edges but about 10\% deeper in the central parts (e.g. Claret \& Hautschildt 2003). Then, we should have obtained $A_{V}=0.009 \mathrm{mag}$ in the $V$-band. A larger discrepancy is present between the amplitudes of OGLE-TR-132 measured by Moutou et al. (2004), $A_{R}=0.0066$, and Udalski et al. (2002), $A_{I}=0.011$. Therefore, given the different methods and the errors, 
we ignore these differences, although it would be interesting to explore (with larger samples of single transits frequently sampled with millimagnitude photometry), if these differences are evidences of systematic effects. In particular, the amplitude measured in the near-infrared would be very useful to test the significance of the differences, even though measuring $A_{K}$ with millimagnitude precision would be a challenge.

The smaller amplitude compared with OGLE results, and the fact that in $V$-band the ingress and egress phases in the light curve are not so steep (Figure 6), make our results differ from those obtained by Silva \& Cruz (2005) for OGLE-TR-109 $\left(R_{p}=1.18 R_{J}\right.$ and $i=89.0)$.

Table 1 summarizes the results obtained from the light curves, giving the period, HJD, transit time, orbital inclination, amplitude, and radius of the companion.

Table 2 lists the previous estimates of the size for the OGLE-TR-109 companion from the literature, and this work. No serious disagreement is found among the four existing measurements of $R_{p}$. In fact, all quoted sizes are substellar, consistent with giant planets or brown dwarfs. As for giant planets is concerned, OGLE-TR-109-b does not seem to be over sized. One would expect that over such close orbit with $a=0.016 \mathrm{AU}$ the planet should be inflated by stellar radiation. If this is indeed the case, we could be in the presence of a very low mass planet.

The companion orbits at a distance of merely $a=0.016 A U=2.4 R_{*}$. Then, the planet is orbiting just outside of the stellar Roche radius. This represents even a more extreme case than those discussed by Patzold, Carona, \& Rauer (2005). They examined the tidal interaction of close-in OGLE planets, concluding that OGLE-TR-56-b, for example, would spin-up the stellar rotation and spiral into the star in a short timescale. It remains to be studied if a similar fate may await OGLE-TR-109-b.

The shortest period planets known are OGLE-TR-56, and OGLE-TR-113 (Udalski et al. 2002, Konacki et al. 2003, Torres et al. 2004a, Bouchy et al. 2004, Konacki et al. 2004). With $P=1.2 d$, and $P=1.4 d$, their orbital periods more than double that of OGLETR-109. If this is a planetary transit, it would be the shortest planet known so far, around the hottest star measured. Unfortunately, the star is rapidly rotating, with $V=35.4 \pm 1.8$ $\mathrm{km} / \mathrm{s}$, which makes the measurement of precise velocities very difficult (Pont et al. 2005). Therefore, in this (and other similar cases that might arise), it is rather important to find other means of establishing the presence of a planet that do not require the measurement of $M_{p} \sin i$ by radial velocities.

One such possibility is to place limits to the ellipsoidal modulation of the light curve due to tidal effects, as discussed by Drake (2003) and Sirko \& Paczynski (2003). A short period 
massive companion would induce a detectable photometric signature in the light curve of the primary star, with a periodicity of half the orbital period. For OGLE-TR-109 Sirko \& Paczynski (2003) measure one of the light curves with least modulations. No ellipsoidal modulation with $P=0.3$ days is detected in our light curve for OGLE-TR-109 larger than $\sim 0.001 \mathrm{mag}$ (Figure 10), supporting their conclusions. A caveat is that our data are not ideal to measure low amplitude modulations of the light curve, as it is well known that contiguous photometric points could be correlated, the OGLE dataset is more useful in this respect.

Drake (2003) modeled the expected amplitudes of the modulation due to ellipsoidal effects for main sequence stars of different spectral types as function of their companion mass. The effect is larger for early type main sequence stars, because their surface gravity is lower than that of a solar-type star, for example. He considered a single period of 2 days, but the modulation increases with decreasing period as $P^{-2}$, and one would expect for his models to provide a strong upper limit for the case of OGLE-TR-109 with $P=0.589$ days. In fact, the effect in the light curve amplitudes for this star should be more than 11 times that shown in Figure 1 of Drake (2003). For example, an F0V star with a $0.1 M_{\odot}$ companion would exhibit a modulation with amplitude of $0.4 \mathrm{mmag}$ if the orbital period is $P=2$ days. For an orbital period of $P=0.589$ days, this amplitude would be about $4.5 \mathrm{mmag}$, certainly not observed in the light curve of OGLE-TR-109. Figure 10 shows the phased light curve of OGLE-TR-109 for nights 2, 3, and 4, along with the expected modulation corresponding to a companion with $M=0.08 M_{\odot}$ (the brown dwarf limit), and $0.045 M_{\odot}$ (the radial velocity limit of Pont et al. 2004). These modulations, with amplitudes 0.0034 and $0.0023 \mathrm{mag}$, respectively, are not seen in our light curve (Figure 10), indicating that the companion mass is smaller than these limits.

The OGLE light curve provides an even more stringent limit to the mass. Figure 11 shows the full OGLE dataset binned in 0.02 phase bins with parts around the transit removed. Typically 30-50 observations were included in each bin. The light curve is folded with the basic period and twice its value (as if the star was an eclipsing binary with equal depth eclipses) - lower and upper panels, respectively. The thick line is an ellipsoidal variation of $1.0 \mathrm{mmag}$ amplitude. It is clear from this plot that the data do not show such variability even at a much lower level. A formal fit to the raw data indicates no significant ellipsoidal variation larger than $0.5 \mathrm{mmag}$ for both periods. This is in good agreement with the result of Sirko \& Paczynski (2003): $a_{c 2}=0.61 \pm 0.33$ mmag. Using the latter amplitude to extrapolate in Figure 1 of Drake (2003) for an F-type main sequence star yields an upper limit of $M=0.014 \pm 0.008 M_{\odot}$ for the companion mass. This rules out a low mass star or brown dwarf, and leaves the possibility of a transiting giant planet. Note that this constraint from the ellipsoidal modulation agrees with the maximum mass limit $M_{\max }=0.045 M_{\odot}$ 
established for the companion by Pont et al. (2005) using radial velocities.

Interestingly, the reflection or heating effect due to the planet might be detectable because of the short orbital axis. The parameter $a_{c 1}$ of Sirko \& Paczynski (2003) measures this effect. In the case of OGLE-TR-109 with $a=0.016$ AU (Silva \& Cruz 2005), the planet with $R=0.9 R_{J}$ intercepts about $1 / 1000$ of the stellar flux, and one would expect $a_{c 1}=1 \mathrm{mmag}$. This is not very different from $a_{c 1}=0.78 \pm 0.35 \mathrm{mmag}$ measured by Sirko \& Paczynski (2003).

\section{A Blend with an Eclipsing Binary?}

Unfortunately, a blend of an F-type main sequence star with a background binary is difficult to rule out. Mandushev et al. (2005) clearly show how a very good transit candidate with consistent radial velocities finally was a blended system. In the absence of radial velocities, the possibility of a blend with a background eclipsing binary star can be explored by detecting secondary transits, by comparing the amplitudes of the individual transits, and by comparing the amplitudes of a single eclipse measured with two different filters.

Alternated eclipses of different depths (i.e. presence of primary and secondary eclipses), would be evidence of an eclipsing binary with period $P=2 \times 0.59=1.18$ days. Note that the eclipses in the first and fourth night do not have high enough quality to compare their amplitudes with the transit shown in Figure 6. However, the OGLE data do not indicate different depth of eclipses within photometric errors.

In addition, the best parts of our light curves in nights 1,2 and 3 monitor the times when the secondary eclipses are expected to happen. No secondary eclipses are detected in the light curves of night 2 (with the smallest scatter), with amplitudes larger than $\sim 0.001$ mag. No secondary eclipses are apparent in the OGLE light curves either.

In some cases a blend with an eclipsing binary should show the heating effects on the secondary, particularly for short period binaries with $P \approx 0.6$ days. However, the reflection effect measured by Sirko \& Paczynski (2003) for OGLE-TR-109 is also small, $a_{c 1}=0.78$, as mentioned above.

At first sight one argument in favor of a blend with an eclipsing binary in the background might the difference between the amplitudes measured in the $V$ and $I$-bands. Udalski et

al. (2002) obtained $A_{I}=0.008 \pm 0.001$. Due to limb darkening, the total transit amplitude in the $V$-band should be about $10 \%$ larger than that, or $A_{V}=0.009 \pm 0.001$. Comparing 
this value with $A_{V}=0.006 \pm 0.001$ measured here, there is a $2.1 \sigma$ difference. This is not significant enough to discard OGLE-TR-109 as a blend.

In order to produce the low amplitude transits, the F0V star is located in the foreground and dominates the flux, while the blend must be with a background main sequence star eclipsed by a late-type main sequence star or a giant eclipsed by a main-sequence star.

If the blend is with a later type main sequence star $(\mathrm{G}$ or $\mathrm{K})$ it would produce $A_{V}$ is larger than $A_{I}$ (e.g. Drake 2003), contrary to the observations. The fact that $A_{V}=0.006 \mathrm{mag}$ is smaller than $A_{I}=0.008 \mathrm{mag}$ would indicate that the binary has a primary star that is fainter and bluer than the F0V star. This scenario must be seriusly considered, because it has been observed in other transiting candidate, OGLE-TR-33, which is a hierarchical triple system composed of a slightly evolved F6 star and an eclipsing binary with a K7-M0 star orbiting an F4 star (Torres et al. 2004b). Only precise radial velocities can rule out (or confirm) this kind of configuration.

The eclipsed red giant scenario is ruled out because of the same reason of the redder background binary, and because it would be bright enough to see in the spectra of OGLETR-109.

An additional piece of evidence against a blend with a redder background binary star is given by Gallardo et al. (2005), who do not detect significant near-infrared excess in the $K$-band. They measured $V-K_{0}=0.51 \pm 0.1$, and $E(B-V)=0.38 \pm 0.02$, adequate for an F0V star in the Milky Way disk.

Another test of binarity or contamination by a background source is to measure the movement of the photocenter during transit with respect to the uneclipsed portions of the light curve. This method, often useful for microlensing events (Alcock et al. 2003), does not yield anything conclusive in this regard because the OGLE-TR-109 transit amplitude is so small.

In order to further explore the blend issue, we have acquired Ks band images with SOFI at the ESO New Technology Telescope in May 2005. Figure 4 shows the same field of view of Figure 2 and 3 in the Ks band. The small circle indicates a very red point source, presumably a late M-type main sequence star that was not present in the V-band images. This source, however, cannot be the source of the TR109 eclipse. There are no other faint red stars within 1 arcsec that would contaminate the photometry of our main target star.

Other sources of variability that may resemble low amplitude transits are star spots. These should not be constant with time, however, as has been observed with the transits of OGLE-TR-109. Furthermore, star spots should vary with the rotation period of the star, 
which in this case should be $P_{\text {rot }} \approx 2$ days, in contradiction with the observations.

To summarize, we have shown that for this special case, the photometry shows no evidence for a blend of the F-type star with a background redder binary, and provides tight enough constraints to discard a massive companion, so OGLE-TR-109-b is most likely a blend between an F-type star and a binary with a bluer primary star or a transiting giant planet.

\section{Conclusions}

Udalski et al. (2002) discovered low amplitude transits in the F0 main sequence star OGLE-TR-109, which we observed with VIMOS at the ESO VLT. The systematic effects dominate over photon noise because OGLE-TR-109 is a relatively bright star. However, we are able to clearly detect the low amplitude transits on this star. We note that this approach is complementary to the one followed by Udalski et al. (2003). They observed 24 transits, but with few points per transit. Here we observe only three transits, but with numerous points during the transit. Given the similar results obtained by the two widely different approaches, it is our strongest conclusion that OGLE-TR-109 is not a false positive transit detection as argued by Pont et al. (2005).

Examining the transit depths and possibility of secondary eclipses, we suggest that the presence of a blend of the F-type star with a redder eclipsing binary is unlikely, but a blend with a binary with a bluer primary star cannot be ruled out by now.

Drake (2003), and Sirko \& Paczynski (2003) showed that it is possible to limit the mass of the secondary using accurate photometry alone. Analyzing the limits to the ellipsoidal variability measured by Sirko \& Paczynski (2003), and the simulations of Drake (2003), we conclude that a low mass stellar companion with $M_{p}>0.014 \pm 0.008 M \odot$ is ruled out, in agreement with the radial velocity limit of $M_{p} \leq 45 M_{J}$ measured by Pont et al. (2005). The precise mass of the planet may remain undecided for quite some time, because the rapid rotation of the primary star precludes the measurement of radial velocities accurate enough to measure the mass of the companion.

Finally, we improve upon the parameters of the transit, in particular measuring a transit time, $t_{T}=1.8 \pm 0.1$ hours, that is in agreement with the orbital and stellar parameters. We also update the ephemerides and orbital parameters of this interesting system, measuring $P=0.58909$ days, $A_{V}=0.006 \pm 0.001 \mathrm{mag}, H J D=2452322.55993$, and $i=77 \pm 5 \mathrm{deg}$.

We have identified two posible scenarios for OGLE-TR-109: a F-type main sequence 
star blended with a binary with a bluer primary star, or a giant planet orbiting with a very short period around a F-type main sequence star. This object would be unique in its class so far. The existence of a low mass companion orbiting an F0V star with $P=0.589$ days pushes not only the limits for planetary formation and migration even further, but also the atmosphere of this object would be beyond the models considered so far. The fate of this object is interesting as well, because tidal interaction may force the accretion by its mother star in a relatively short timescale.

DM, JMF, GP, MZ, MTR, WG are supported by Fondap Center for Astrophysics No. 15010003. DM also thanks the John Simon Guggenheim Foundation. Partial support to the OGLE project was provided by the Polish MNII grant 2P03D02124, NSF grant AST-0204908 and NASA grant NAG5-12212. A.U. acknowledges support from the grant "Subsydium Profesorskie" from the Foundation for Polish Science. We thank the ESO staff at Paranal Observatory.

\section{REFERENCES}

Alard, C. 2000, A\&ASuppl., 144, 363

Alard, C., \& Lupton, J. 1998, ApJ, 503,325

Alcock, C., et al. 2000, ApJ, 542, 281

Bouchy, F., et al. 2004, A\&A, 421, L13

Claret, A., \& Hautschildt, P. 2003, A\&A, 412, 241

Drake, A. J. 2003, ApJ, 589, 1020

Gallardo, J., Minniti, D., Valls-Gabaud, D., \& Rejkuba, M. 2005, A\&A, 431, 707

Gaudi, S. A. 2005, ApJ, in press (astro-ph/0504123)

Kasting, J. F., Whitmire, D. P., \& Reynolds, R. T. 1993, Icarus, 101, 108

Peeper, J., \& Gaudi, S. A. 2005, ApJ, in press (astro-ph/0504162)

Konacki, M., Torres, G., Jha, S., Sasselov, D. D. 2003, Nature, 421, 507

Konacki, M., Torres, G., Sasselov, D. D., Pietrzynski, G., Udalski, A., Jha, S., Ruiz, M. T., Gieren, W., \& Minniti, D. 2004, ApJ, 609, L37

Mandushev, G.,et al. 2005, ApJ, 621, 1061

Moutou, C., et al. 2005, A\&A, 424, L31

Patzold, M., Carone, H., \& Rauer, H. 2005, A\&A, 427, 1075 
Pont, F., et al. 2005, A\&A, 438, 1123

Schneider, J. 2005, The Extrasolar Planets Encyclopaedia

Silva, A. V. R., \& Cruz, P. C. 2005, ApJ in press (astro-ph/0505281)

Sirko, E., \& Paczynski, B. 2003, ApJ, 592, 1217

Torres, G., Konacki, M., Sasselov, D., \& Jha, S. 2004a, ApJ, 609, 1071

Torres, G., Konacki, M., Sasselov, D., \& Jha, S. 2004b, ApJ, 614, 979

Udalski, A., Szewczyk, O., Zebrun, K., Pietrzynski, G., Szymanski, M., Kubiak, M., Soszynski, I., Wyrzykowski, L. 2002, Acta Astronomica, 52, 317

Udalski, A., Pietrzynski, G., Szymanski, M., Kubiak, M., Zebrun, K., Soszynski, I., Wyrzykowski, L. 2003, Acta Astronomica, 53, 133

Zahn, J.-P. 1977, A\&A, 57, 383 
Table 1. Measured Parameters for OGLE-TR-109-b

\begin{tabular}{lll}
\hline \hline \multicolumn{1}{c}{ Parameter } & \multicolumn{1}{c}{ Value } & \multicolumn{1}{c}{ Comment } \\
\hline$P(\mathrm{~d})$ & 0.589128 & \\
$J D_{0}(\mathrm{~d})$ & $2452322.55993 \pm 0.001$ & \\
Transit duration $(\mathrm{hr})$ & $1.8 \pm 0.1$ & \\
transit amplitude $(\mathrm{mag})$ & $0.006 \pm 0.001$ & \\
Inclination (deg) & $77 \pm 5$ & Pont et al. $(2004)$ \\
Companion radius $R_{p}\left(R_{J}\right)$ & $0.90 \pm 0.09$ & \\
Companion mass $\left(M_{J}\right)$ & $<45$ & \\
Companion mass $\left(M_{J}\right)$ & $<14 \pm 8$ & \\
\hline
\end{tabular}

Table 2. Radius of OGLE-TR-109-b

\begin{tabular}{lll}
\hline \hline \multicolumn{1}{c}{ Reference } & \multicolumn{1}{c}{$R_{p}\left(R_{J}\right)$} & \multicolumn{1}{c}{ Comments } \\
\hline Udalski et al. (2002) & 0.99 & lower limit, 24 transits, $I$-band photometry \\
Gallardo et al. (2005) & $1.31 \pm 0.10$ & using OGLE amplitude, new stellar parameters \\
Silva \& Cruz (2005) & 1.18 & re-analysis of the OGLE $I$-band photometry \\
This work & $0.90 \pm 0.09$ & single transit, $V$-band photometry \\
\hline
\end{tabular}




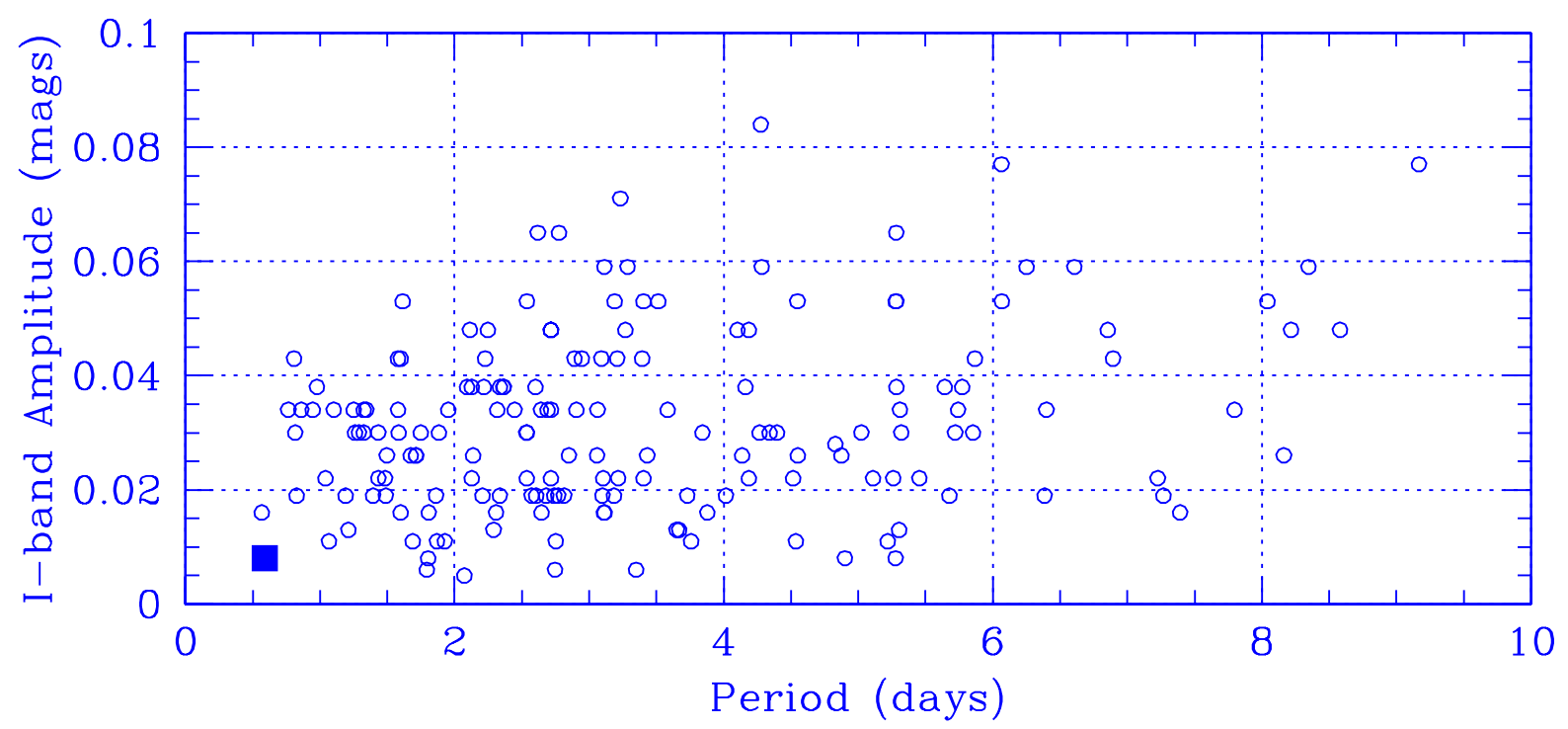

Fig. 1.- Amplitudes and periods of the OGLE transit candidates. The star OGLE-TR-109 is indicated with the solid square. 


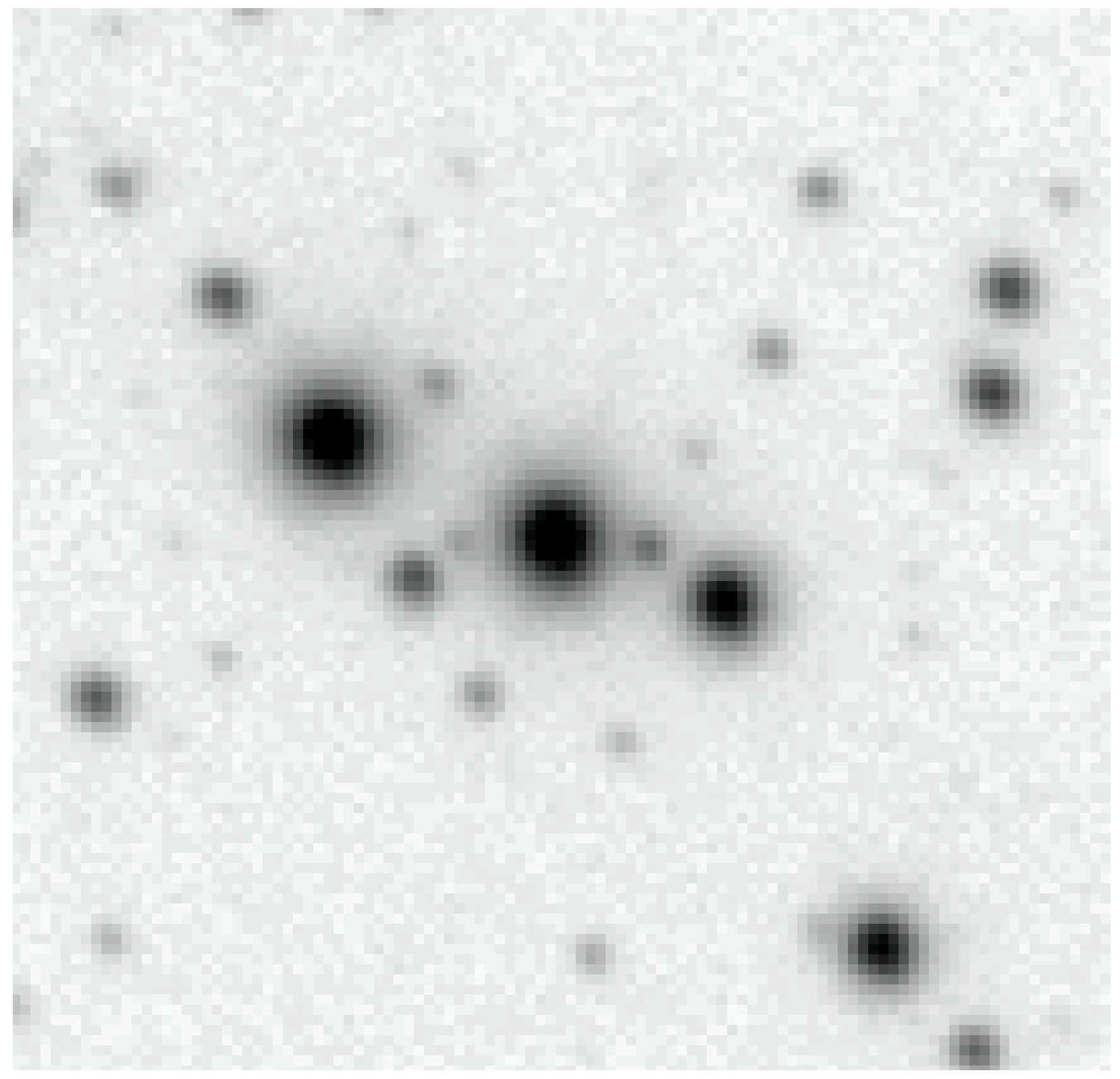

Fig. 2.- Finding chart obtained with VIMOS for OGLE-TR-109 $(V=15.80)$, which is the bright star at the center. This image covers $20 \times 20$ arcsec, and represents the best FWHM obtained $(0.5$ arcsec). The faintest stars seen in this image have $V \sim 24$. 


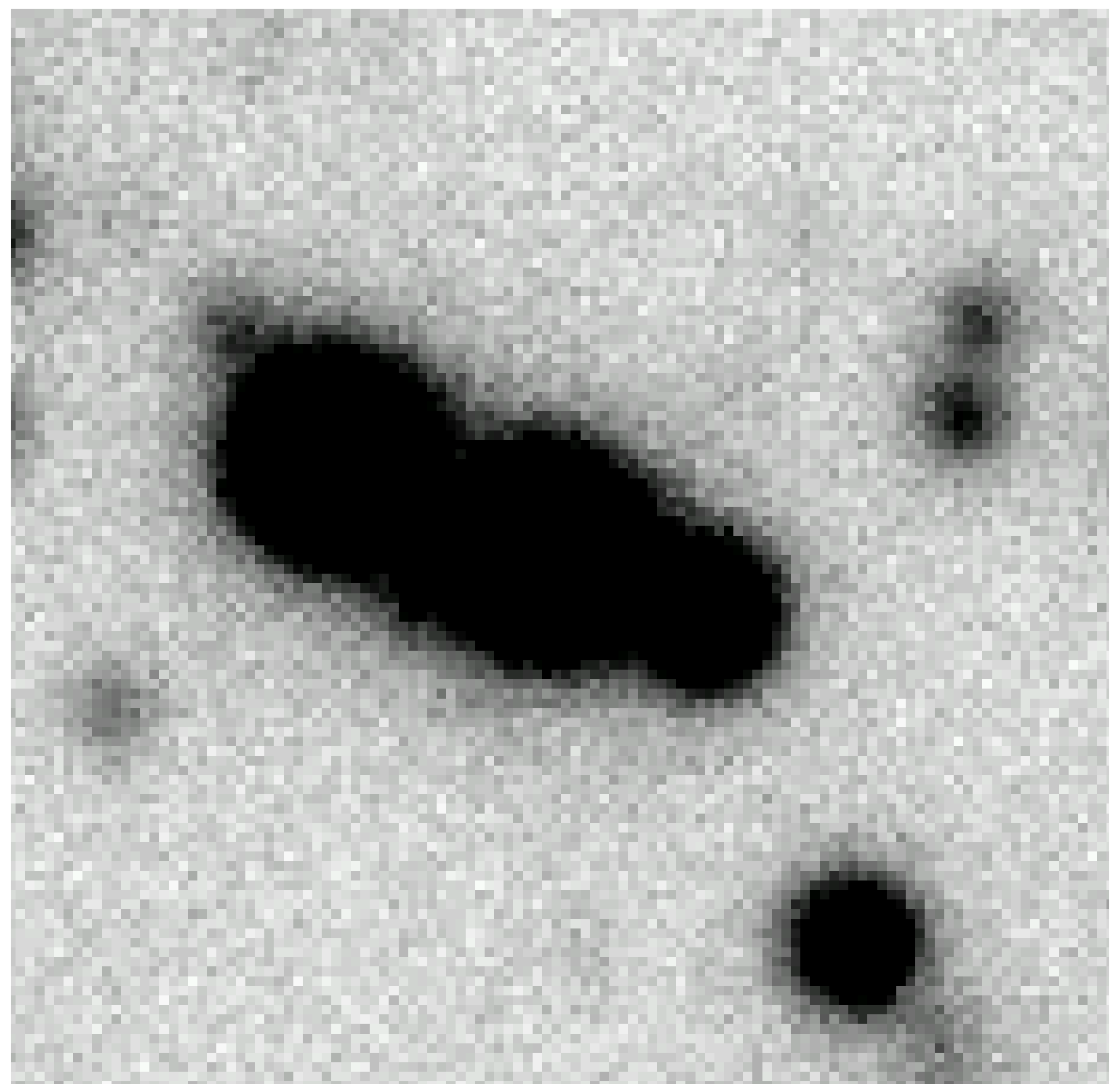

Fig. 3.- Same as Figure 2. This image covers $20 \times 20$ arcsec, and represents the worst FWHM obtained $(1.6$ arcsec) at high airmass $(X \sim 3)$. The faintest stars seen in this image have $V \sim 20$. There is clear blending of the flux from the two bright companion stars to OGLE-TR-109. Even using the difference image analysis, these images yield very poor photometry for our target $(r m s \sim 0.02)$, rendering them useless when trying to measure the transits to millimagnitude accuracy. 


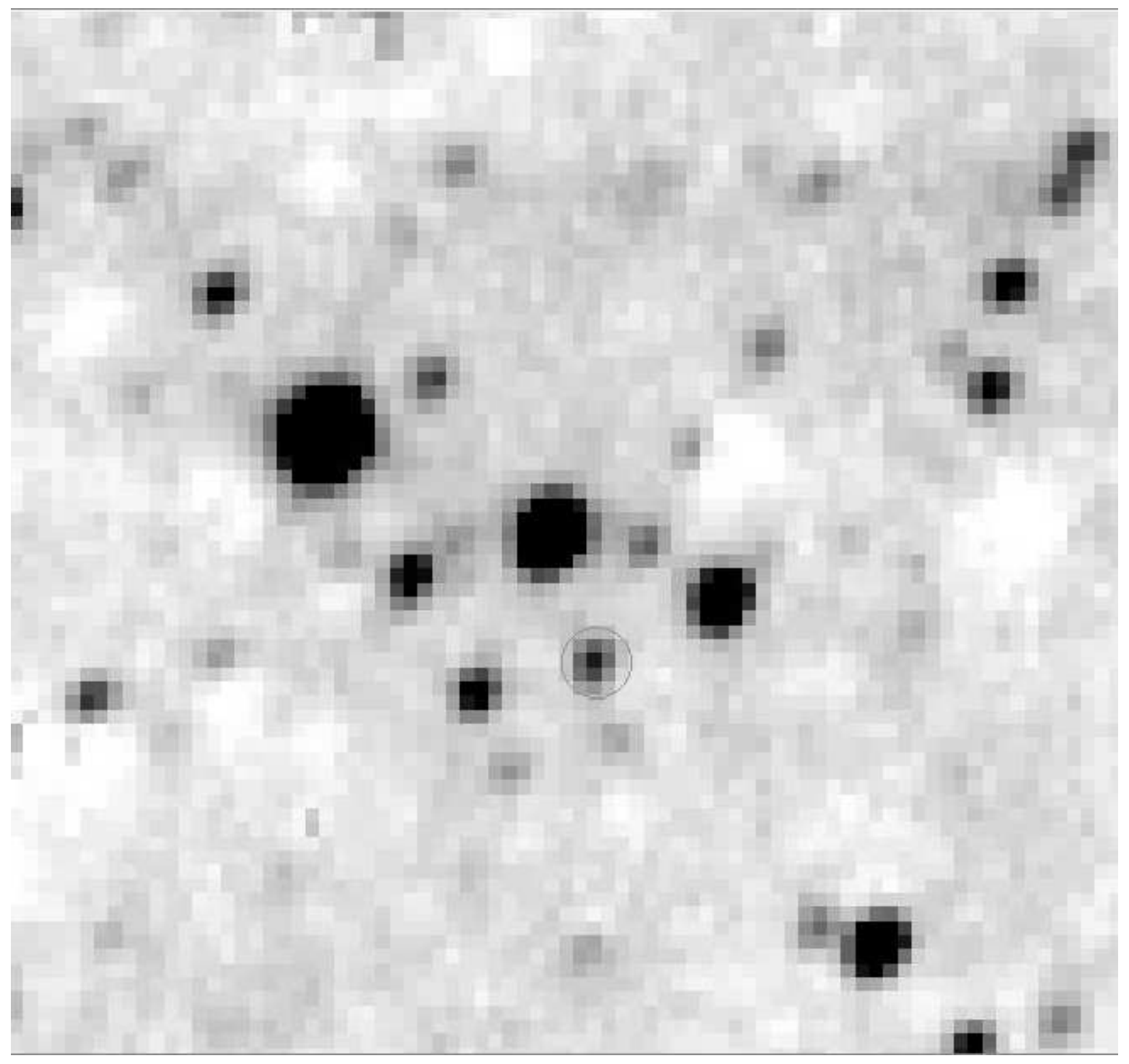

Fig. 4.- Same field of view of Figure 2 and 3 in the Ks band. The small circle indicates a very red point source, presumably a late M-type main sequence star that was not present in the V-band images. 


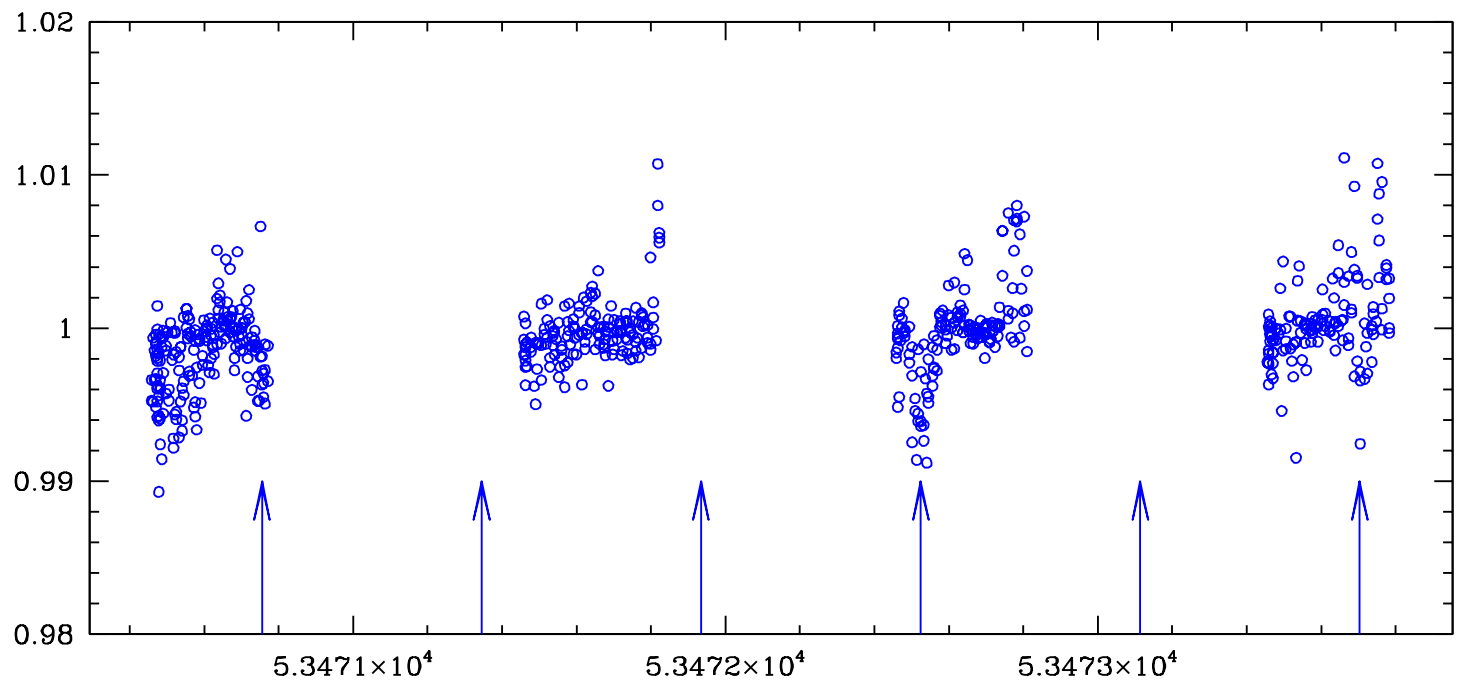

Fig. 5.- Light curve of OGLE-TR-109 during the four nights. All 650 points measured are plotted, including points obtained at high airmass at the end of the nights, for which the diminished quality of the photometry is obvious. The third night shows a well monitored transit. The predicted mean transit times from OGLE are shown with the arrows.

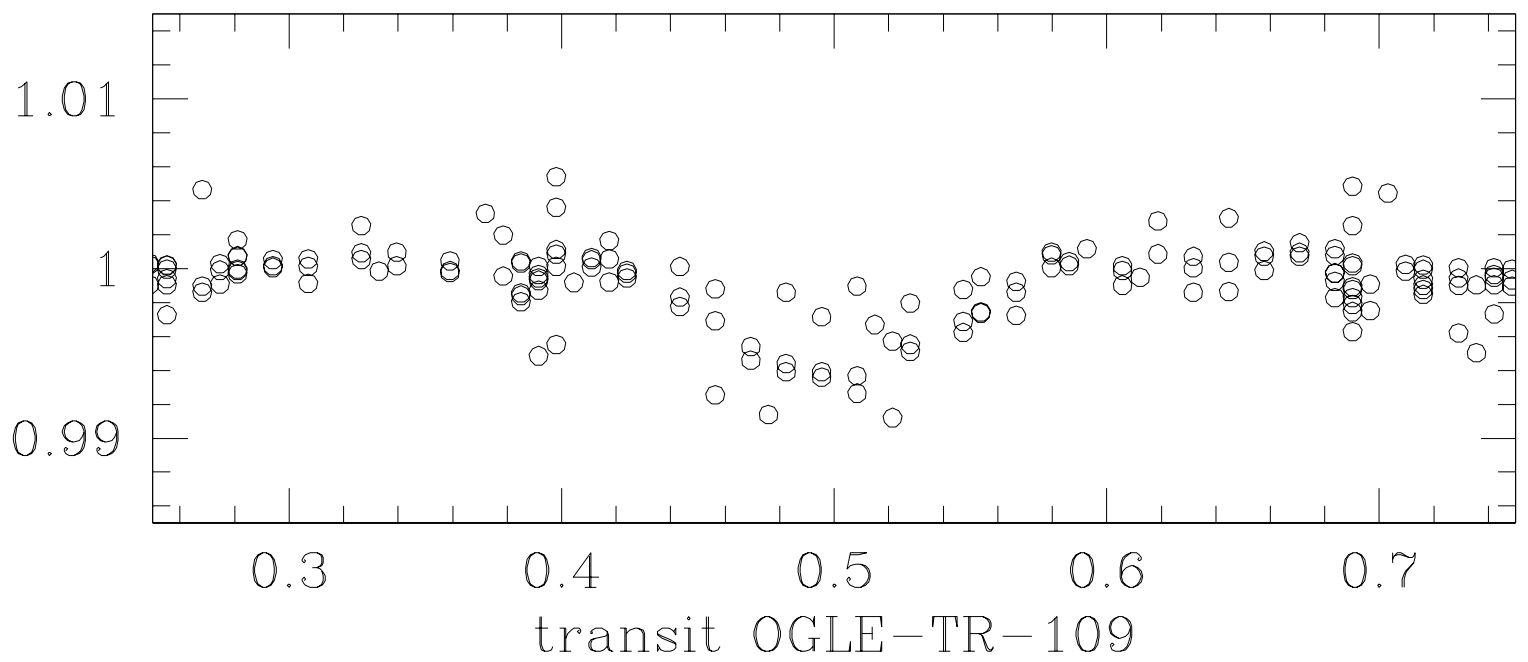

Fig. 6. - Best single transit of OGLE-TR-109 observed during the third night with VIMOS. 


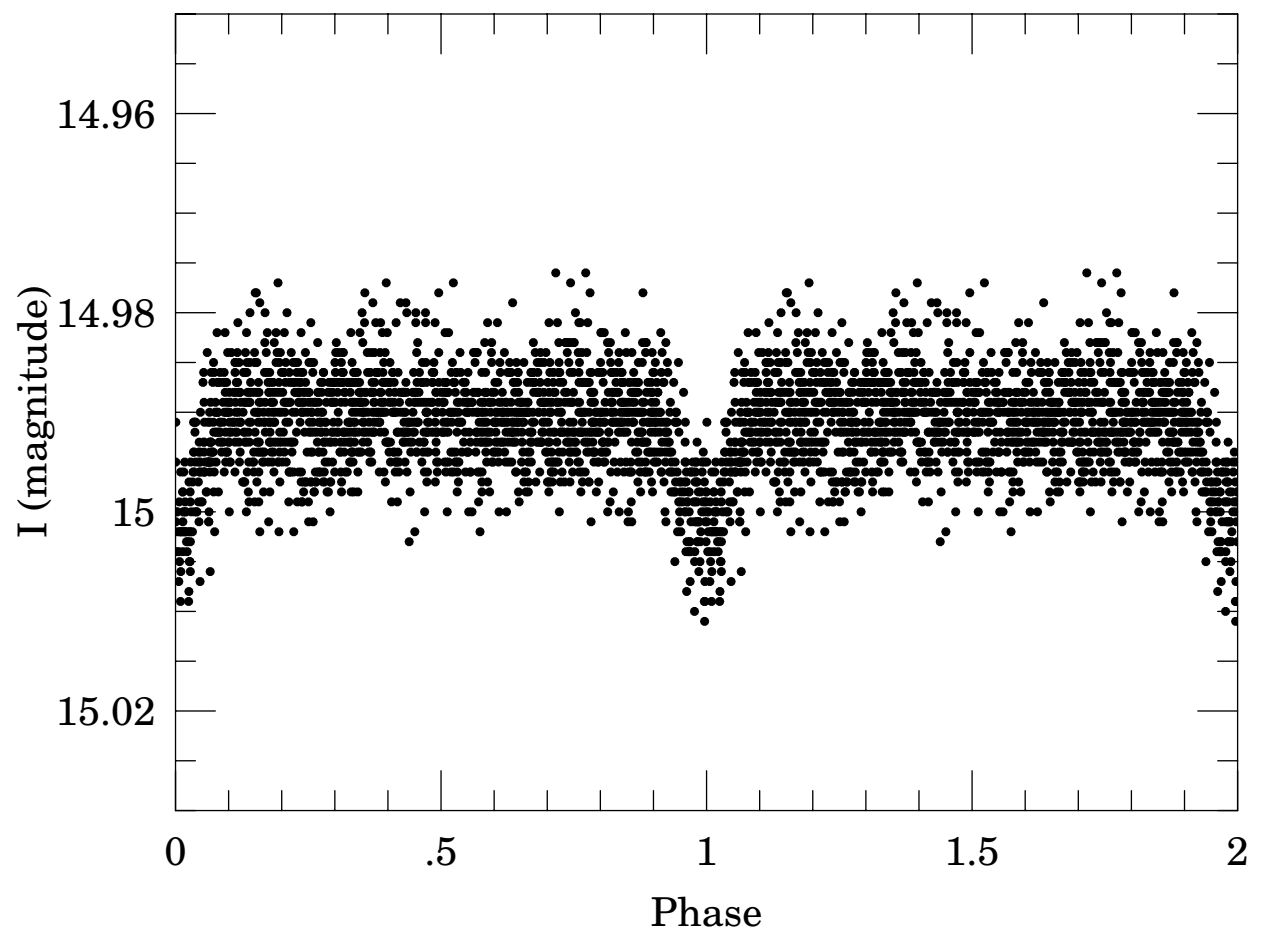

Fig. 7.- New phased light curve of OGLE-TR-109 for the OGLE data in the $I$-band. 


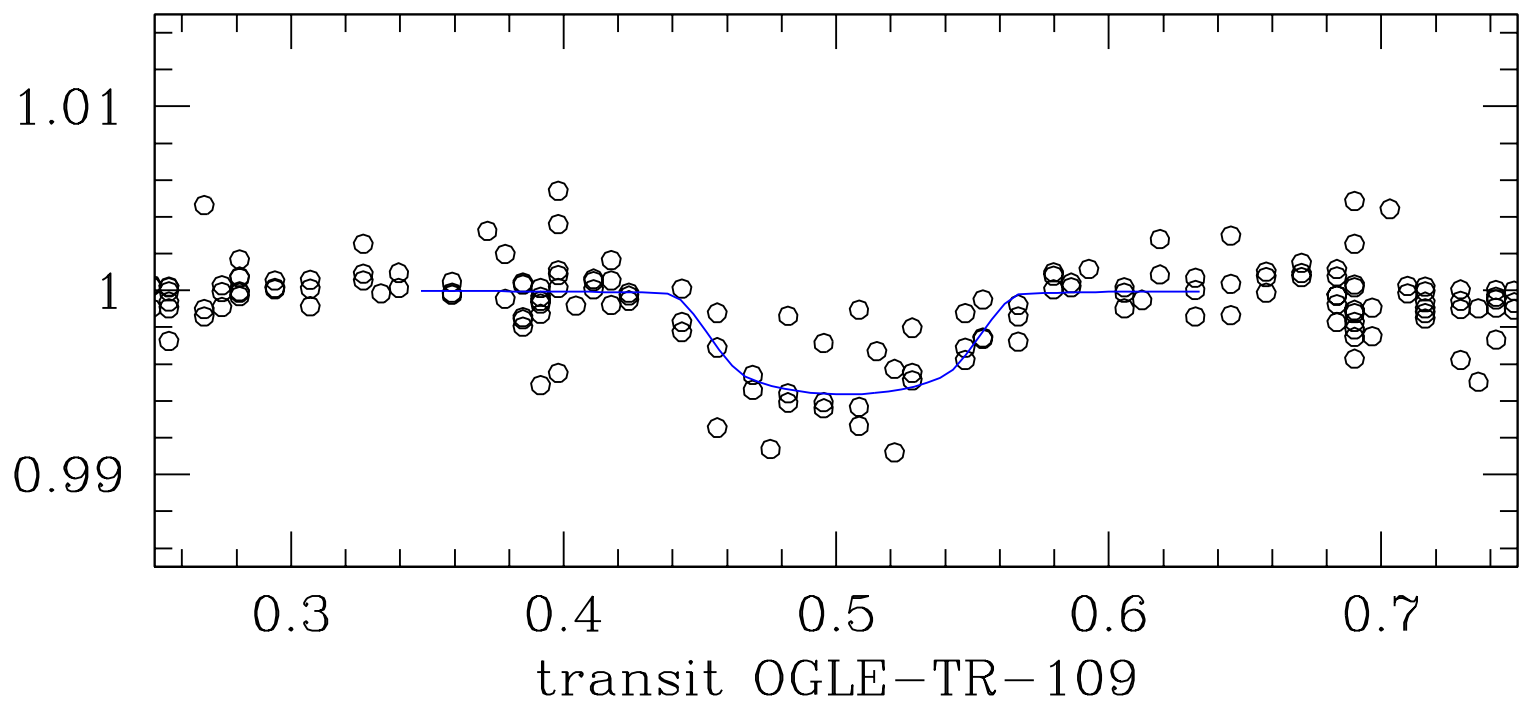

Fig. 8. - Fit to the best single transit of OGLE-TR-109 observed during the third night with VIMOS. 


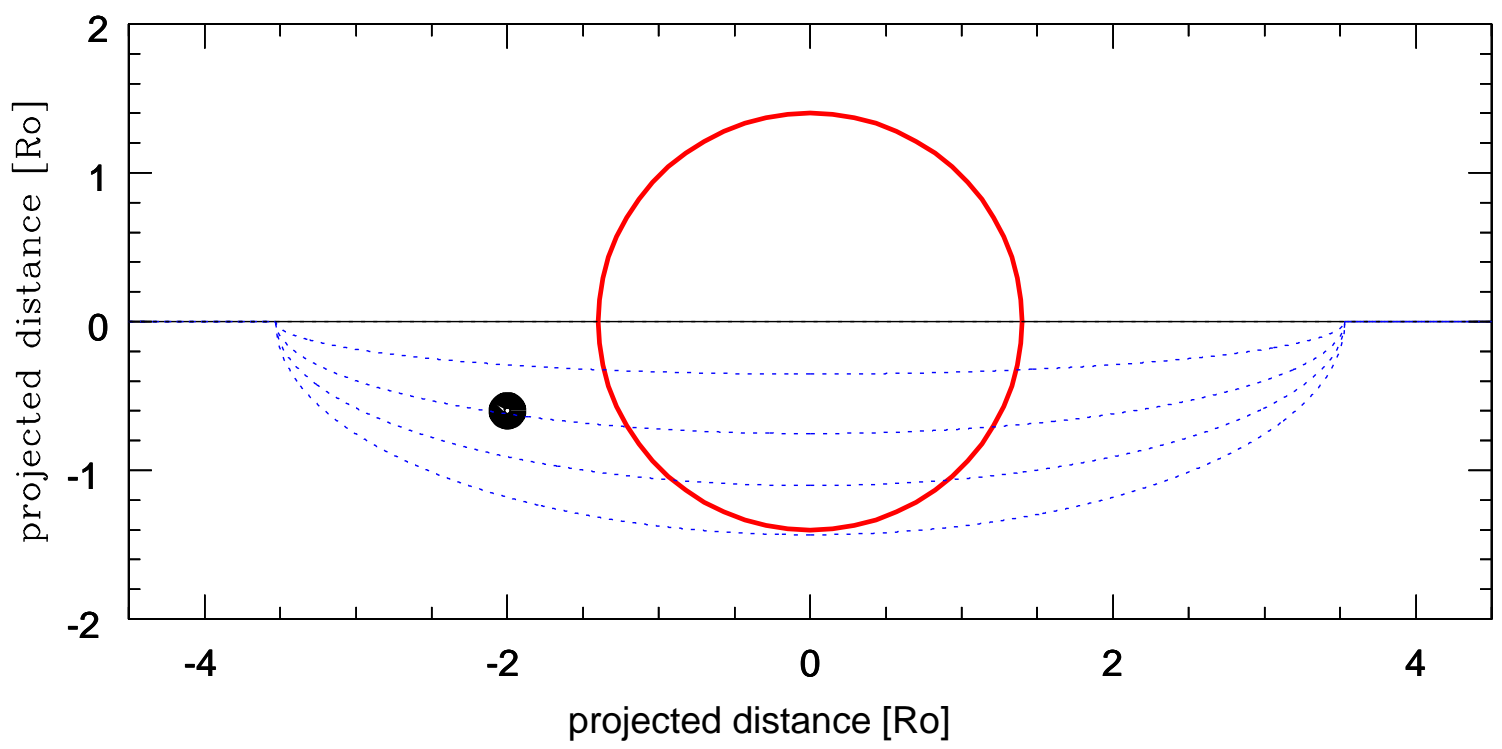

Fig. 9.- Forefront half of the OGLE-TR-109 orbit (and transit configuration) drawn to scale for different inclination angles: $i=90,84,78,72$, and 66 degrees, from top to bottom. The smaller inclinations are ruled out because the measured transit duration is too long for a grazing eclipse. The larger inclinations are ruled out because of the inclined ingress and egress phases. Note that the trajectory in front of the star departs from linearity because of the short orbital axis. 


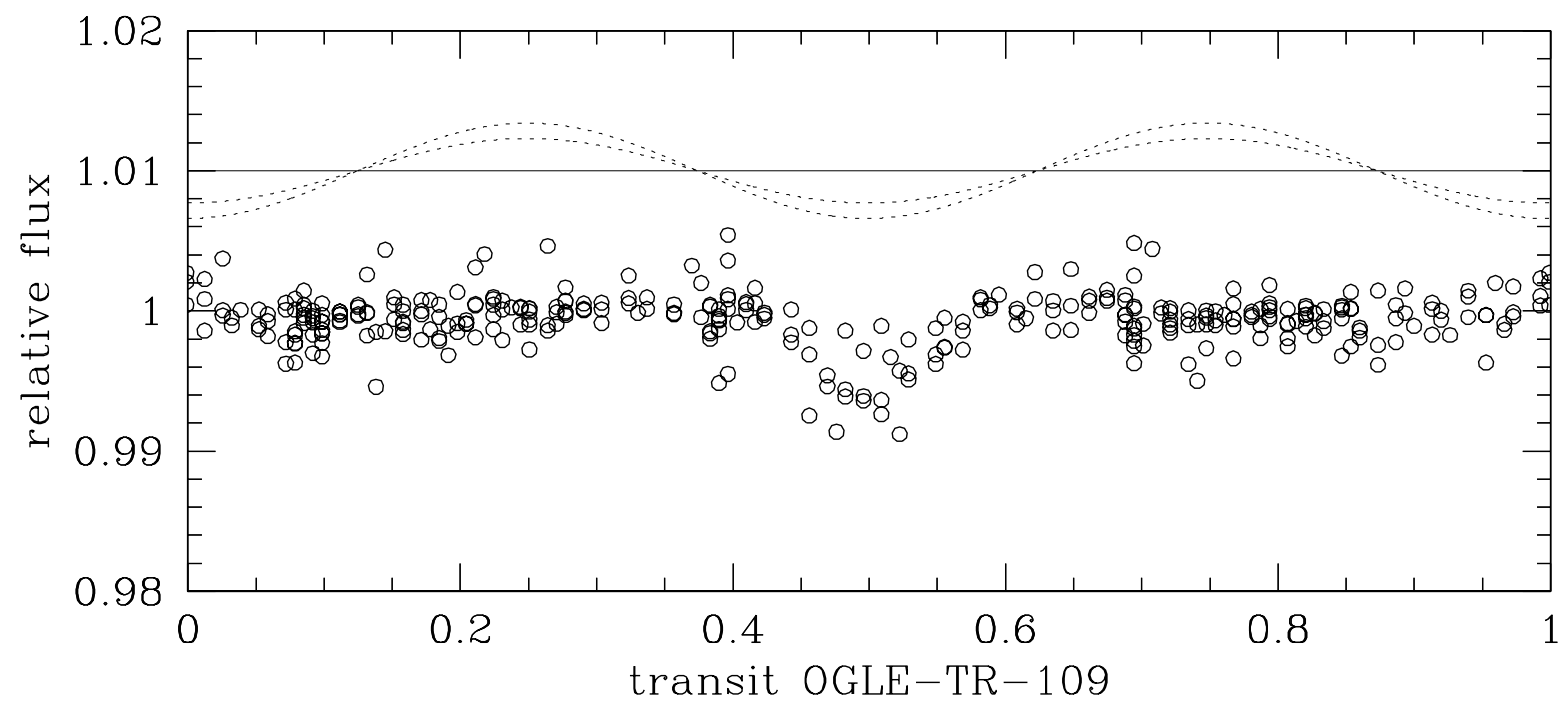

Fig. 10.- Phased light curve of OGLE-TR-109 for nights 2, 3, and 4. For comparison, the expected ellipsoidal modulation corresponding to a companion with $M=0.08$ and $0.045 M_{\odot}$ are shown with the dashed lines above the light curve, with amplitudes 0.0034 and 0.0023 mag, respectively. The fact that no such light modulations are seen allows to constrain the mass of the companion. 


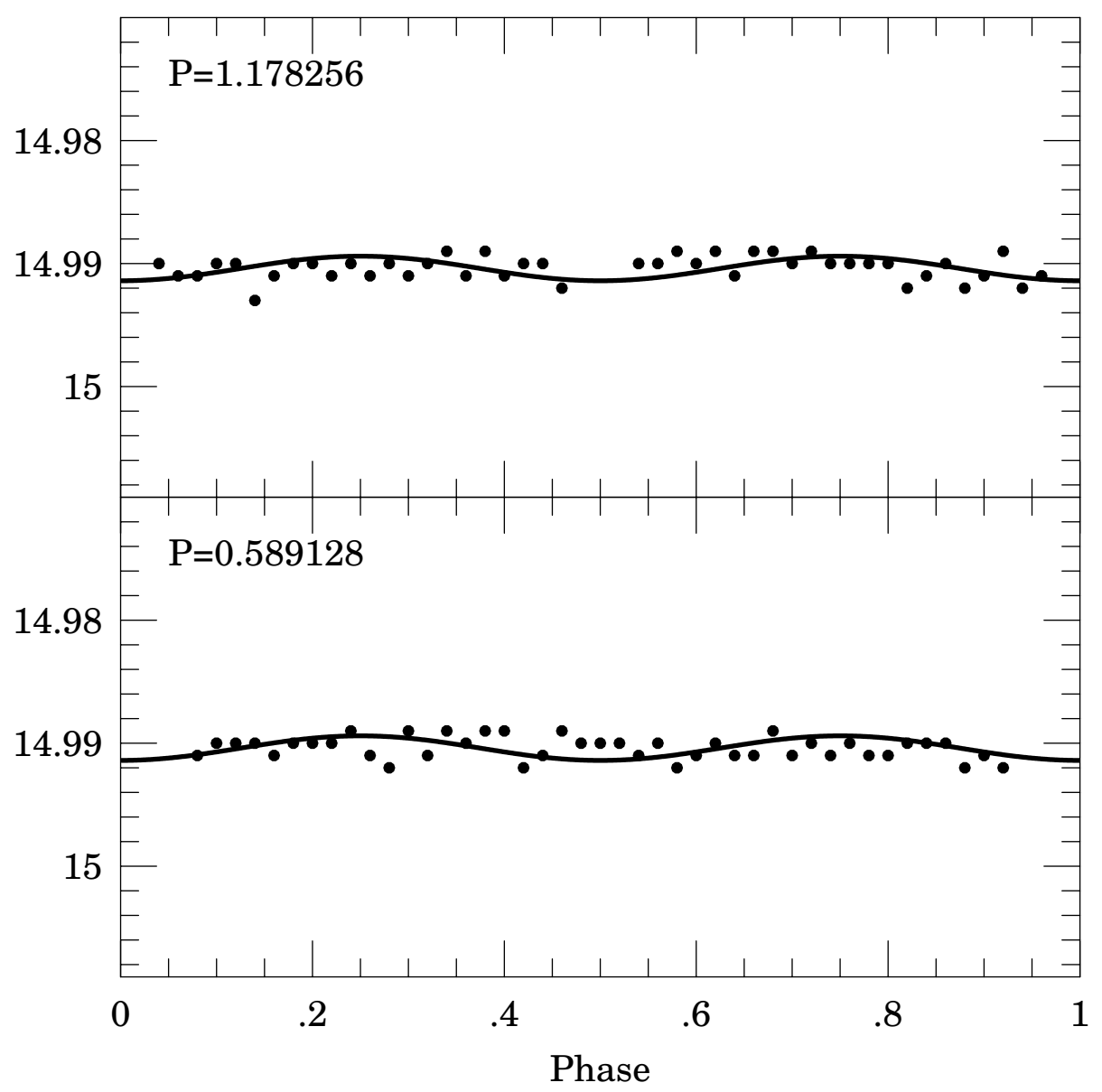

Fig. 11. - Light curve of OGLE-TR-109 for the OGLE data in the $I$-band, binned in 0.02 phase bins with parts around the transit removed. The light curve is folded with the basic period and twice its value. The thick lines show the expected ellipsoidal modulation with amplitude of $1.0 \mathrm{mmag}$. 